\title{
An Improved Graph-Entropy Bound for Perfect Hashing *
}

Erdal Arikan

Electrical Engineering Department, Bilkent University, 06533 Ankara, Turkey

\section{Abstract}

We give an improved graph-entropy bound on the size of families of perfect hash functions. Examples are given illustrating that the new bound improves previous bounds in several instances.

Perfect hashing is a method of information storage and retrieval [1]. It is also equivalent to certain zero-error list-coding problems of information theory [4]. Following [3], call a set of sequences of length $t$ over a $b$-letter alphabet $k$-separated if for every $k$-tuple of sequences there exists a coordinate in which they all differ. Let $N(t, b, k)$ denote the largest possible size for such a set of sequences. Perfect-hashing is the problem of finding such maximal sets. Here, we give an upper bound on the asymptotic quantity (the capacity)

$$
C_{b, k}:=\limsup _{t \rightarrow \infty} \frac{1}{t} \log N(t, b, k)
$$

(Logarithms are to base 2.) This bound extends earlier results given in [5] and is based on a refinement of the graph-entropy bound $[2]-[4]$.

Körner and Marton [3] show that

$$
C_{b, k} \leq \min _{0 \leq j \leq k-2} \frac{b^{j+1}}{b^{j+1}} \log \frac{b-j}{k-j-1}
$$

where $b^{i}=b(b-1) \cdots(b-i+1)$. We give here the bound

$$
C_{b, k} \leq \sup \{x: x \leq \alpha,(x), j=2, \ldots, k-2\}
$$

where

$$
\alpha_{j}(x)=\frac{b-j}{k-1} 2^{-x}\left(1-\frac{x}{\log b}\right) \frac{b^{j}}{b^{j}} \log \frac{b-j}{k-1-j}
$$

for $j=2, \ldots, b-k$ and

$$
\alpha_{j}(x)=\left(1-\frac{j}{b-k+1}\left(1-2^{-x}\right)\right)\left(1-\frac{x}{\log b}\right) \frac{b^{j}}{b^{j}} \log \frac{b-j}{k-1-j}
$$

for $j=b-k+1, \ldots, k-2$.

The following table lists the values of the new bound (2) and the Körner-Marton bound (1). The integers in parentheses indicate the values of $j$ which optimize the corresponding bounds. The table demonstrates that the new bound improves the earlier graph-entropy bound in many instances. To our knowledge, the values in the table constitute the best available bounds on $C_{b, k}$

\begin{tabular}{|c|c|c|c|}
\hline$b$ & $k$ & New Bound & KM Bound \\
\hline \hline 4 & 4 & $0.3511(2)$ & $0.3750(2)$ \\
5 & 4 & $0.6114(2)$ & $0.7370(0)$ \\
5 & 5 & $0.2359(3)$ & $0.1920(3)$ \\
6 & 4 & $0.8390(2)$ & $1.0000(0)$ \\
6 & 5 & $0.4414(3)$ & $0.4402(3)$ \\
6 & 6 & $0.1548(4)$ & $0.0925(4)$ \\
7 & 4 & $1.029(2)$ & $1.2223(0)$ \\
7 & 5 & $0.6204(3)$ & $0.6997(3)$ \\
7 & 6 & $0.3055(4)$ & $0.2376(4)$ \\
7 & 7 & $0.0974(5)$ & $0.0428(5)$ \\
100 & 6 & $3.6184(2)$ & $4.3219(0)$ \\
100 & 10 & $2.830(2)$ & $3.3219(0)$ \\
\hline
\end{tabular}

\section{References}

[1] M. Fredman and J. Komlós, 'On the size of separating systems and perfect hash functions,' SIAM J. Algebraic and Discrete Methods, vol. 5, no. 1, pp. 61-68, 1984.

[2] J. Körner, 'Fredman-Komlós bounds and information theory,' SIAM J. Algebraic and Discrete Methods, vol. 7, no. 4 , pp. $560-570$, Oct. 1986 .

[3] J. Körner and K. Marton, 'New bounds for perfect hashing via information theory,' Europ. J. Combinatorics, vol. 9 , pp. $523-530,1988$.

[4] J. Körner and K. Marton, 'On the capacity of uniform hypergraphs,' IEEE Trans. Inform. Theory, vol. IT-36, No.1, pp. 153-156, Jan. 1990.

[5] E. Arikan, 'An upper bound on the zero-error list-coding capacity,' Proceedings of 1993 IEEE Int. Symp. Inform. Theory, p. 152, San Antonio, USA, Jan 17-22, 1993.

*This research was supported by TÜBITAK under project TBAG 1053 